\title{
Fisioterapia en el syndrome de Smith-Magenis: a propósito de un caso
}

\author{
Physiotherapy in Smith-Magenis syndrome: a case report
}

\author{
M. González Leiro, M. Chouza-Insua, F.J. Senín-Camargo, S. Viñas Diz
}

\begin{abstract}
Resumen
El síndrome de Smith-Magenis es una alteración del desarrollo psicomotor de origen genético, incluido en el grupo de enfermedades raras. En el caso clínico que se presenta, se realizó una valoración inicial de la coordinación, la marcha y el equilibrio a través del test de desarrollo psicomotor (TEPSI), un circuito diseñado específicamente, la prueba del tablero de clavijas de Purdue y el test Timed up and Go. Al finalizar los 3 meses que duró el tratamiento, basado en actividades de tipo lúdico, se realizó una valoración final con las mismas pruebas, encontrándose importantes diferencias objetivas en los resultados obtenidos, además de una mejoría subjetiva reportada por los padres del paciente. A pesar de que la evidencia sobre el tratamiento de fisioterapia en este síndrome es escasa, atendiendo a los resultados encontrados creemos que dicho tratamiento está totalmente justificado en el abordaje integral de los pacientes que lo padecen.
\end{abstract}

\begin{abstract}
The Smith-Magenis syndrome is a genetic psychomotor development disorder included in the group of rare diseases. In the clinical case presented, we performed an initial assessment of coordination, gait and balance using four different tests. These were psychomotor development test (TEPSI scale), Purdue pegboard, time up and go test, and a circuit designed specifically for this clinical case. At the end of the 3 months of treatment, and based on recreational type activities, a final assessment was made with the same tests. Significant objectives differences in the results as well as subjective improvement reported by the patient's parents were found. Although there is little evidence on physiotherapy treatment for this syndrome, in accordance with the results found, we believe that this treatment is totally justified in the comprehensive approach to patients suffering this disorder.
\end{abstract}

Palabras clave

Fisioterapia; Enfermedad rara; Psicomotricidad

Keywords

Physiotherapy; Rare disease; Psychomotricity

\section{Introducción}

Según diversas normativas de la Unión Europea, como el Reglamento (CE) n. ${ }^{\circ}$ 141/2000, las enfermedades raras se definen como aquellas enfermedades poco comunes, incluidas las de origen genético, que pueden ser mortales o provocar un debilitamiento crónico del paciente y que, debido a su escasa prevalencia, requieren esfuerzos combinados para tratarlas. A título indicativo, se considera una prevalencia escasa cuando es inferior a 5 casos por cada 100.000 personas en la comunidad. Una de estas enfermedades raras es el síndrome de Smith-Magenis (SMS), que presentamos en este caso clínico. El SMS fue descrito por primera vez en 1982 por Ann Smith ${ }^{1}$ y se caracteriza por la pérdida de un pequeño fragmento intersticial del cromosoma 17 , concretamente en la banda G17p11.2, lo que sugiere un bajo riesgo de recurrencia para los padres ${ }^{2}$. El origen se sitúa en el gen ácido inducido retinoico 1 (Rai1) o por una mutación del mismo ${ }^{3}$. Esta 
alteración genética va a producir un trastorno complejo caracterizado por un retraso mental variable acompañado por otras características, como trastornos del sueño, anomalías craneofaciales y esqueléticas, comportamientos autolesivos y de búsqueda de atención, junto con retraso motor y en el habla ${ }^{4}$. El SMS afecta por igual a ambos sexos, con una incidencia de uno de cada 25.000 nacidos vivos en el mundo, con un rango de entre 15.000 y 25.000 , pudiendo ser esta una subestimación, ya que si la deleción es pequeña puede llegar a perderse en la rutina del análisis citogenético 5 . Debido a esta baja incidencia, este síndrome es aún poco conocido tanto en el ámbito sanitario (hospitalario y atención primaria) como en el ámbito educativo y en la comunidad en general.

Los métodos y las técnicas propios de la fisioterapia pueden ser de utilidad en enfermedades, cuadros clínicos o síndromes que conlleven un retraso en el desarrollo psicomotor; por lo tanto, estarán indicados en pacientes de SMS, para abordar tanto las alteraciones del desarrollo psicomotor como las demás características presentes en este cuadro clínico. El objetivo de este estudio es el de comprobar si el tratamiento combinado psicomotricidad/fisioterapia mejora la calidad de vida del sujeto.

\section{Caso clínico}

El paciente abordado es un varón de 11 años de edad, diagnosticado de SMS. En el examen inicial, se recogieron los siguientes datos.

Estudia en un colegio no adaptado, acompañado de un cuidador. Visita una vez a la semana al psicólogo y al logopeda. Presenta buena comprensión y expresión oral, es un niño muy hablador. Presta mucha atención a estímulos auditivos. Baja estatura para su edad $(1,29 \mathrm{~m})$. No presenta alteraciones del sueño. Padece astigmatismo, aunque no suele usar gafas porque intenta romperlas.

Se observa una deficiencia de la motricidad fina: presenta dificultad para realizar actividades con ambas manos, especialmente con la mano izquierda que suele estar más cerrada y con una posición mantenida de flexión palmar.

Presenta dificultad a la hora de hacer transiciones de una actividad a otra; los cambios inesperados generan crisis en su comportamiento. A menudo repite preguntas o comentarios de forma recurrente, insistiendo mucho en el mismo tema, lo que podría estar relacionado con problemas de memoria a corto plazo.

Usa vocabulario negativo y provocador, para atraer nuestra atención. Y muestra una conducta compulsiva-disruptiva.

\section{Tratamiento}

Se realizó una sesión de fisioterapia por semana en el ámbito escolar, durante 3 meses. Cada sesión tuvo una duración de 45-60 min en función del cansancio del paciente y se llevó a cabo en un aula de educación infantil del colegio al que asiste habitualmente el niño.

El tratamiento se centró en mejorar las habilidades motoras (gruesas y finas), el equilibrio y la marcha, a través de actividades con una importante orientación lúdica, que resultasen motivadoras para el niño:

- Actividades en mesa orientadas a la mejora de la motricidad fina:

- Actividades en diferentes posiciones (bipedestación, sedestación en balón suizo, en cuadrupedia y de rodillas) orientadas al entrenamiento de la coordinación, equilibrio y marcha.

- Actividades de recepción y lanzamiento de pelotas de diferentes tamaños.

- Circuito (descrito en la fig. 1).

- Usamos un refuerzo positivo (consensuado con el psicólogo) como estrategia motivacional, dejando para el final de la sesión uno de los juegos que más le motiva y satisface. 


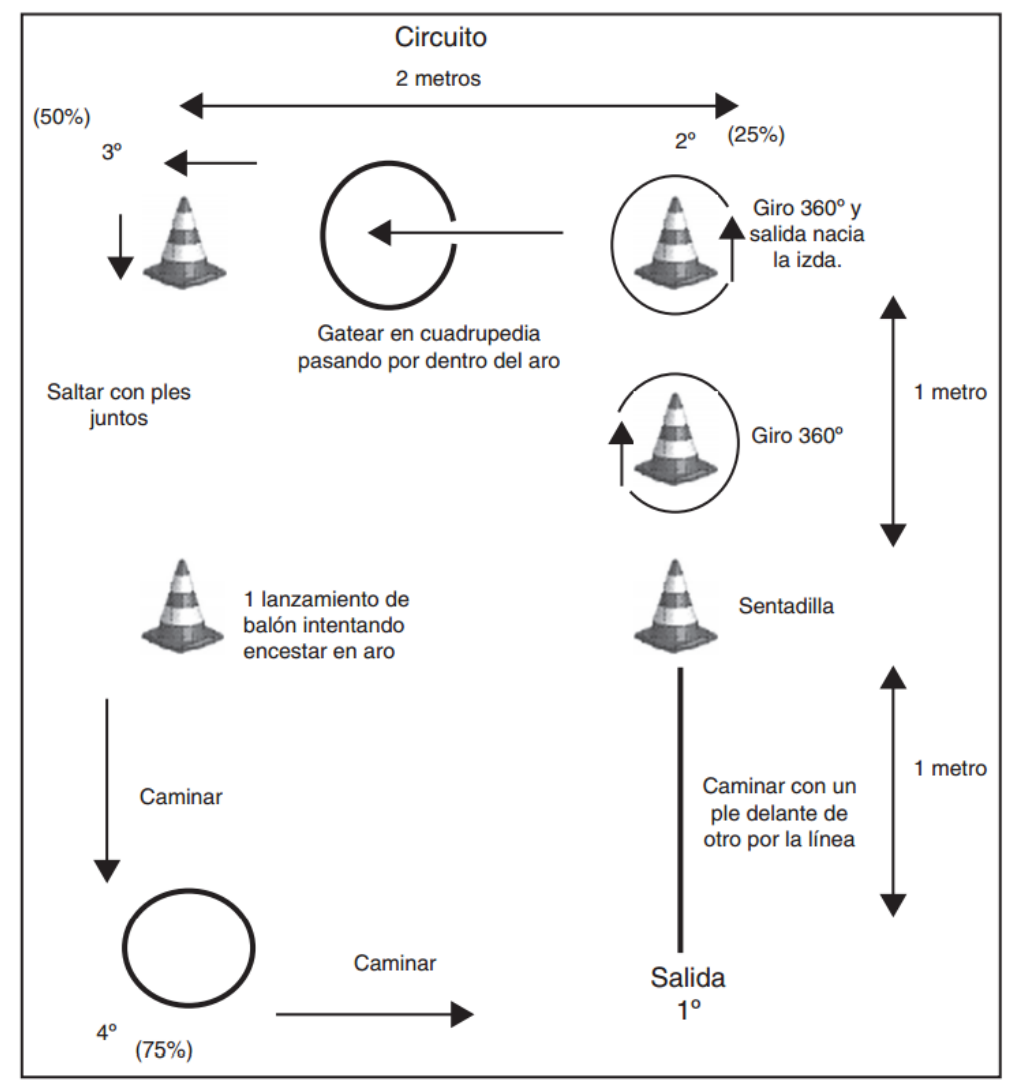

Figura 1. Circuito específicamente diseñado para el paciente.

Antes de iniciar cada sesión se retiran de su alcance todos los objetos que puedan lastimar o dañar, ya que puntualmente, en un momento de crisis puede intentar agredir a terceras personas (tira del pelo, da manotazos, intenta meter dedo en el ojo o incluso puede intentar morder las manos al sujetarlo) o autolesionarse.

Durante la sesión se dan al paciente instrucciones claras y fáciles (p. ej., tírame la pelota despacio), junto con recordatorios regulares para evitar su inquietud y distracción, y así pueda completar la actividad. Intentamos potenciar su autocontrol, exigiendo en la realización de las actividades, que controle la fuerza, la velocidad de los movimientos, etc. También intentamos mantener un mismo orden en las sesiones, para favorecer la rutina durante las mismas, evitando así comportamientos indeseados ante la dificultad que le supone las transiciones entre actividades. Ante la repetición constante de una misma pregunta, empleamos estrategias como devolverle la pregunta, mostrar indiferencia o hacer una señal visual que puede hacer referencia a lo mismo. En el momento en el que utilice un vocabulario negativo o provocador, no debe notarnos alterados o molestos; se ignoran los insultos u otras palabras que use con sentido negativo, para que dejen de tener sentido para él.

\section{Resultados}

Se realizó una valoración funcional de la coordinación, la marcha y el equilibrio; para ello se ejecutaron 2 mediciones, una antes de iniciar el tratamiento y otra después de 3 meses de comenzado el mismo (tabla 1). Las pruebas empleadas fueron las siguientes: 
- TEPSI (apartado de coordinación y motricidad fina) ${ }^{6}$.

- $\quad$ Test «Timed up and Go» ${ }^{7}$.

- Circuito específicamente diseñado para el paciente (fig. 1).

- $\quad$ Prueba del tablero de clavijas de Purdue (motricidad fina) ${ }^{8}$.

Tabla 1. Resultados de las pruebas realizadas antes y después de recibir el tratamiento

\begin{tabular}{|c|c|c|c|}
\hline Prueba específica de valoración & Valoración inicial & Valoración final (3 meses) & $\begin{array}{l}\text { Diferencia } \\
\text { final-inicial }\end{array}$ \\
\hline $\begin{array}{l}\text { Tablero Purdue (mano derecha)-30 } \\
\text { segundos }\end{array}$ & 6,67 (media 3 mediciones) & 8,67 (media 3 mediciones) & 2 \\
\hline $\begin{array}{l}\text { Tablero Purdue (mano izquierda)-30 } \\
\text { segundos }\end{array}$ & 6,3 (media 3 mediciones) & 6,67 (media 3 mediciones) & 0,37 \\
\hline TEPSI (subtest coordinación) & 9 puntos & 13 puntos & 4 puntos \\
\hline TEPSI (subtest motricidad) & 6 puntos & 10 puntos & 4 puntos \\
\hline Timed up and Go & $\begin{array}{l}\text { 11,35 segundos (media } 3 \\
\text { mediciones) }\end{array}$ & $\begin{array}{l}8,3 \text { segundos (media } 3 \\
\text { mediciones) }\end{array}$ & $\begin{array}{c}-3,05 \\
\text { segundos }\end{array}$ \\
\hline Circuito (n. ${ }^{\circ}$ vueltas en $\left.1 \mathrm{~min}\right)$ & 1 vuelta; $75 \%$ de la 2. avuelta & $\begin{array}{l}2 \text { vueltas; } 50 \% \text { de la } \\
\text { 3.a vuelta }\end{array}$ & $\begin{array}{l}0,75 \% \text { de } \\
\text { vuelta }\end{array}$ \\
\hline
\end{tabular}

Los resultados obtenidos, indican que el paciente mejoró su funcionalidad.

\section{Discusión y conclusiones}

En general, es escasa la información publicada sobre el SMS; podemos encontrar publicaciones en las que se recoge a modo descriptivo las alteraciones que componen el fenotipo clínico y ofrecen posibilidades de tratamiento de tipo farmacológico y psiquiátrico ${ }^{\text {and }} 10$. Otros artículos se centran en aspectos de tipo genómico y pruebas diagnósticas $11^{\text {and }} 12$, algo comprensible teniendo en cuenta que se trata de un síndrome cuya base es una alteración genética concreta y, como en el resto de perturbaciones de este tipo, la comunidad científica necesita especificar y aislar la secuencia alterada para así poder descubrir y detectar nuevos casos mediante los diversos procedimientos diagnósticos. No hay datos publicados sobre la evidencia de la fisioterapia en el SMS; sin embargo, el cuadro clínico que presentan los pacientes con este síndrome permite justificar el empleo de la misma como parte del tratamiento interdisciplinar, ya que se trata de un síndrome donde está presente una compleja alteración que afecta no solo al ámbito físico, sino también al ámbito psíquico y al desarrollo psicomotor del individuo.

La buena evolución observada en este paciente ratifica el importante papel de la fisioterapia en el SMS. A pesar del complicado abordaje, el tratamiento integral de estos pacientes es imprescindible para mejorar la realización de las actividades de la vida diaria de una manera más eficaz y autónoma, así como mejorar la calidad de vida, tanto del individuo como de las personas que están a su cargo. 


\section{Responsabilidades éticas}

Protección de personas y animales. Los autores declaran que para esta investigación no se han realizado experimentos en seres humanos ni en animales.

Confidencialidad de los datos. Los autores declaran que en este artículo no aparecen datos de pacientes.

Derecho a la privacidad y consentimiento informado. Los autores han obtenido el consentimiento informado de los pacientes y/o sujetos referidos en el artículo. Este documento obra en poder del autor de correspondencia.

\section{Bibliografía}

1. A.C.M. Smith, L. McGavran, G. Waldstein. Deletion of the 17 short arm in two patients with facial clefts. Am 7 Hum Genet, 34 (1982), p. 410A

2. O. Udwin, C. Webber, I. Horn. Abilities and attainment in Smith-Magenis syndrome. Dev Med Child Neurol, 43 (2001), pp. 823-828

3. R.E. Slager, T.L. Newton, C.N. Vlangos, B. Finucane, S.H. Elsea. Mutations in RAI1 associated with Smith-Magenis syndrome. Nat Genet, 33 (2003), pp. 466-468

4. S.H. Elsea, S. Girirajan. Smith-Magenis syndrome. Eur J Hum Genet, 16 (2008), pp. 412-421

5. E. Sweeney, I. Peart, M. Tofeig, B. Kerr. Smith-Magenis syndrome and tetralogy of fallot. J Med Genet, 36 (1999), pp. 501-502

6. I.M. Heussler, T. Marchant. Tepsi. Test de desarrollo psicomotor 2-5 años. Ediciones Universidad Católica de Chile, Santiago (2003)

7. D. Podsiadlo, S. Richardson. The timed 'Up \& Go': a test of basic functional mobility for frail elderly persons. J Am Geriatr Soc, 39 (1991), pp. 142-148

8. J. Tiffin, E.J. Asher. The Purdue pegboard: norms and studies of reliability and validity. J Appl Psychol, 32 (1948), pp. 234-247

9. E.A. Edelman, S. Girirajan, B. Finucane, P.I. Patel, J.R. Lupski, A.C.M. Smith, et al. Gender, genotype, and phenotype differences in Smith-Magenis syndrome: a meta-analysis of 105 cases. Clin Genet, 71 (2007), pp. 540-550

10. P.L. Wolters, A.L. Gropman, S.C. Martin, M.R. Smith, H.L. Hildenbrand, C.C. Brewer, et al. Neurodevelopment of children under 3 years of age with Smith-Magenis syndrome Pediatr Neurol, 41 (2009), pp. 250-258

11. S. Girirajan, C.N. Vlangos, B.B. Szomju, E. Edelman, C.D. Trevors, L. Dupuis, et al. Genotypephenotype correlation in Smith-Magenis syndrome: evidence that multiple genes in $17 \mathrm{p} 11.2$ contribute to the clinical spectrum. Genet Med, 8 (2006), pp. 417-427

12. T. Yamamoto, H. Ueda, M. Kawataki, M. Yamanaka, T. Asou, Y. Kondoh, et al. A large interstitial deletion of 17p13.1p11.2 involving the Smith-Magenis chromosome region in a girl with multiple congenital anomalies. Am J Med Genet, 140 (2006), pp. 88-91 